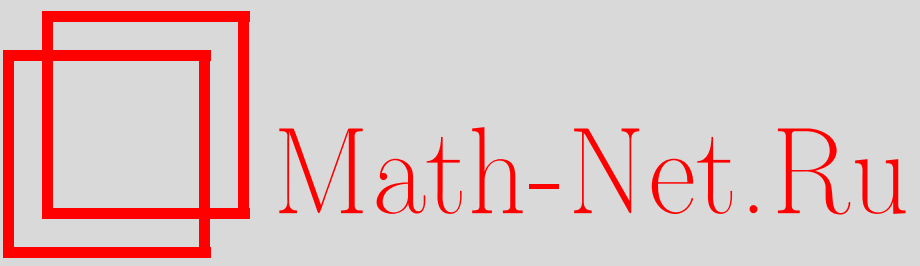

А. В. Пухликов, Бирациональные автоморфизмы трехмерных алгебраических многообразий, расслоенных на кубические поверхности, УМН, 1997, том 52, выпуск $1,235-236$

DOI: https://doi.org/10.4213/rm814

Использование Общероссийского математического портала Math-Net.Ru подразумевает, что вы прочитали и согласны с пользовательским соглашением

http://www. mathnet.ru/rus/agreement

Параметры загрузки:

IP : 54.162 .27 .143

26 апреля 2023 г., 13:56:07 


\title{
БИРАЦИОНАЛЬНЫЕ АВТОМОРФИЗМЫ ТРЕХМЕРНЫХ АЛГЕБРАИЧЕСКИХ МНОГООБРАЗИЙ, РАССЛОЕННЫХ НА КУБИЧЕСКИЕ ПОВЕРХНОСТИ
}

\author{
А. В. Пухликов
}

1. В настоящей работе дан набросок доказательства (уточненной) гипотезы В. А. Исковских об алгебраических многообразиях с пучком кубических поверхностей [1], являющейся точным аналогом известной теоремы В. Г. Саркисова о расслоениях на коники. В. А. Исковских предположил, что она может быть доказана методом максимальных особенностей [2] и сделал первые шаги в ее доказательстве. Однако далшше продвинуться не удалось в силу недостатков техники. Они были преодолены в работе [3] для пучков поверхностей Дель Пеццо степени 1 и 2. Данная работа является ее непосредственным продолжением.

2. Пусть $V$ - гладкое трехмерное комплексное проективное многообразие, обладающее морфизмом $\pi: V \rightarrow \mathbb{P}^{1}$, каждый слой которого-неприводимая приведенная кубическая поверхность, а общий слой $F_{\eta}$ - гладкая кубическая поверхность над незамкнутым полем $\mathbb{C}\left(\mathbb{P}^{1}\right)$ с группой Пикара $\operatorname{Pic} F_{\eta}=\mathbb{Z} K_{F_{\eta}}$. В частности, Pic $V=\mathbb{Z} K_{V} \oplus \mathbb{Z} F$, где $F$ - слой морфизма $\pi$. Предположим, что многообразие $V$ удовлетворяет следующему требованию [3]: класс 1-циклов $M K_{V}^{2}-f$, где $f$ - класс прямой в слое, не является эффективным ни для какого $M \in \mathbb{Z}$, а также следующему условию общности положения: $(*)$ если слой $F_{t}=\pi^{-1}(t)$ над точкой $t \in \mathbb{P}^{1}$ - особая кубическая поверхность в $\mathbb{P}^{3}$, то он имеет единственную невырожденную особую точку $q \in F_{t}$. Тогда имеет место

Теорема. Любое бирациональное отображение

$$
\chi: V \rightarrow V^{\prime},
$$

где $V^{\prime}$ - неособое в коразмерности 1 трехмерное проективное многообразие, расслоенное морфизмом $\pi^{\prime}: V^{\prime} \rightarrow \mathbb{P}^{1}$ на поверхности отричательной кодаировой размерности, является послойньм, т.е. $\pi^{\prime} \circ \chi=\alpha \circ \pi$ для некоторого изоморфизма базы $\alpha: \mathbb{P}^{1} \rightarrow \mathbb{P}^{1}$.

СледСтВИя. (i) $V$ обладает единственным пучком рачиональных поверхностей. $B$ частности, $V$ не рационально и не имеет структуры расслоения на коники.

(ii) Факторгруппа группь $\operatorname{Bir} V$ бирациональных автоморфизмов многообразия $V$ по нормальной подгруппе бирачиональных автоморфизмов, сохраняющ, слои $\pi$, изоморфной группе $\operatorname{Bir} F_{\eta}$ бирациональных автоморфизмов общего слоя, конечна, в общем случае тривиальна.

ЗАмЕчАния. (i) Относително обильный пучок $\mathscr{O}_{V}\left(-K_{V}\right)$ порождается на каждом слое своими сечениями и определяет двойное вложение $V \hookrightarrow X$, где $X=\mathbb{P}(\mathscr{E}), \mathscr{E}=\mathscr{O} \oplus \mathscr{O}\left(a_{1}\right) \oplus$ $\mathscr{O}\left(a_{2}\right) \oplus \mathscr{O}\left(a_{3}\right)$ - локально свободный пучок ранга 4 на $\mathbb{P}^{1}, 0 \leqslant a_{1} \leqslant a_{2} \leqslant a_{3}$, совпадающий с точностью до подкручивания на обратимьй пучок с $\pi_{*} \mathscr{O}_{V}\left(-K_{V}\right)$. Проекцию $X$ на $\mathbb{P}^{1}$ снова обозначаем символом $\pi$. Пусть $G$-класс слоя морфизма $\pi: X \rightarrow \mathbb{P}^{1}$, тогда $\operatorname{Pic~} X=\mathbb{Z} L \oplus \mathbb{Z} G$, где $L$ - класс тавтологического пучка $\mathscr{O}_{X / \mathbb{P}^{1}}(1)$, т.е. $\pi_{*} \mathscr{O}_{X}(L)=\mathscr{E}$. Предположим, что $V \sim 3 L+m G$ как дивизор на $X$. Легко проверить, что (основное) условие теоремы выполнено при $5 m+3\left(a_{1}+\right.$ $\left.a_{2}+a_{3}\right) \geqslant 12$. В частности, если $X=\mathbb{P}^{1} \times \mathbb{P}^{3}$, т.е. $a_{1}=a_{2}=a_{3}=0$, то получаем условие $m \geqslant 3$, т.е. $V$ задается уравнением $p_{3, m}\left(x_{0}, x_{1}, x_{2}, x_{3} ; u, v\right)=0$ бистепени $(3, m), m \geqslant 3$, см. [1] и [4], где нерациональность общего (в комплексно-аналитическом смысле) многообразия $V \subset \mathbb{P}^{1} \times \mathbb{P}^{3}$ доказана методом вырождения Клеменса.

(ii) Условие общности положения $(*)$ является излишним. Теорема справедлива и без него. Однако количество частных случаев, требующих отдельного рассмотрения, в общем случае существенно больше, и соответствующие выкладки не доведены автором до конца.

(iii) Группа бирациональных автоморфизмов общего слоя описана в [5].

Работа выполнена при финансовой поддержке Российского фонда фундаментальных исследований (грант № 96-01-00820). 
3. Схема доказательства теоремы. Пусть $|\chi|$ - собственный прообраз пучка слоев морфизма $\pi^{\prime}$ относительно $\chi$. Сушествуют однозначно определенные числа $n=n(\chi) \in \mathbb{Z}_{+}$и $l \in \mathbb{Z}_{+}$ такие, что $|\chi| \subset\left|-n(\chi) K_{V}+l F\right|$. Если $n(\chi)=0$, то $l=1$ и утверждение теоремы выполнено. В противном случае ситуация оказьвается противоречивой. Если $n(\chi) \geqslant 1$, то $l \geqslant 0$. В самом деле, пучок $|\chi|$ не имеет неподвижных компонент. Пусть $D_{1}, D_{2} \in|\chi|$ - общие дивизоры, тогда $Z=\left(D_{1} \cdot D_{2}\right)$ есть эффективньй 1-цикл, класс которого есть $n^{2} K_{V}^{2}+2 n l\left(-K_{V}\right.$. $F)=n^{2} K_{V}^{2}+2 n l d f$. В силу сделанного предположения $l$ не может быть отрицателшным. Далее, пучок $|\chi|$ обладает максимальной особенностью [1]-[3] - нормированием $\nu \in \mathscr{N}(V)$ таким, что $e(\nu)=\nu(|\chi|)-n \delta(\nu)>0$, где $\delta(\nu)=K(V, \nu)$ - каноническая кратность (дискрепантность). Более того, можно показать, что либо существует максималная особенность $\nu$ такая, что ее центр - прямая на $V$, либо существует конечное множество максимальных особенностей $\mathscr{M} \subset \mathcal{N}(V)$, центры которых $Z(V, \nu)=x(\nu)$ - точки на $V$, причем выполнено неравенство

$$
\sum_{t \in \mathbb{P}^{1}}\left(\max _{\left\{\nu \in \mathscr{M} \mid x(\nu) \in F_{t}=F_{\nu}\right\}} \frac{e(\nu)}{\nu\left(F_{t}\right)}\right)>l .
$$

Максимальные кривые исключаются либо откручиваются по обычной схеме [1], [5], поэтому можно считать, что реализуется второй случай. Назовем неприводимую кривую $C \subset V$ горизонтальной, если $\pi(C)=\mathbb{P}^{1}$, и вертикальной, если $\pi(C)$ - точка. Эффективньй 1-цикл $Z=\left(D_{1} \cdot D_{2}\right), D_{1}, D_{2} \in|\chi|$, запишем в виде

$$
Z=Z^{v}+Z^{h}=\sum_{t \in \mathbb{P}^{1}} Z_{t}^{v}+Z^{h},
$$

где $Z^{h}$ - горизонтальная, $Z^{v}$ - вертикальная части $Z$, а $Z_{t}^{v}$ - та часть $Z^{v}$, которая содержится в слое $F_{t}$. Из выписанного выше неравенства можно вывести, что найдется максимальная особенность $\nu$, центр которой - точка $x \in F_{t}$ такая, что $6 n e(\nu)>\nu\left(F_{t}\right) \operatorname{deg} Z_{t}^{v}$, где степень понимается в смысле антиканонической линейной системы этого слоя. Дальнейшая работа строится по следующему плану. Если через точку $x$ не проходит ни одна прямая, лежащая на $F_{t}$, то нетрудно показать, что для любой кривой $C \subset F_{t}$ выполнено неравенство mult $x=\frac{2}{3} \operatorname{deg} C$. Теперь,

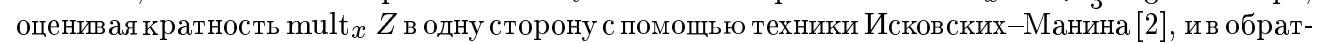
ную сторону через степень накрытия $\pi: Z^{h} \rightarrow \mathbb{P}^{1}$ и $\operatorname{deg} Z_{t}^{v}$, так, как это сделано в [3] (и с учетом вьписанного выше неравенства), получаем противоречие, доказьвающее нашу теорему. Однако самшім трудньм является случай, когда через неособую точку $x \in F_{t}$ проходит одна или несколько прямых (если точка $x$ - особая на слое $F_{t}$, то ситуация упрощается). Здесь метод, развитый в [3], не работает. Однако учет вклада, который дает прямая $L \ni x$ в 1 -цикл $Z_{t}^{v}$, позволяет получить противоречие и в этом случае. Соответствующий анализ является весьма тонким и требует рассмотрения всех бесконечно близких базисных кривых линейной системы $|\chi|$, накрьвающих прямую $L$. Подробности будут опубликованы в другом месте.

\section{СПИСОК ЛИТЕРАТУРЫ}

[1] Исковских В. А. // Труды МИАН. 1995. Т. 208. С. 128-138. [2] Исковских В. А., Манин Ю. И. // Матем. сб. 1971. Т. 86. № 1. С. 140-166. [3] Pukhlikov A. V. Birational automorphisms of Del Pezzo fibrations // Warwick Preprint, 1996. [4] Bardelli F. // Ann. Mat. Pura Appl. (4). 1984. V. 137. Р. 287-369. [5] Манин Ю. И. Кубические формы. М.: Наука, 1972. 